Pathologe 2011 · 32:357-359

DOI 10.1007/s00292-011-1522-3

Online publiziert: 18. August 2011

(c) Springer-Verlag 2011
H.H. Kreipe

Institut für Pathologie, Medizinische Hochschule Hannover

\title{
Rezeptornachweis beim Mammakarzinom
}

\section{Zehn Jahre Ringversuche in der deutschsprachigen Pathologie}

Im Juli dieses Jahres wurde zum zehnten Mal ein neuer Ringversuch zum Nachweis von Östrogenrezeptor, Progesteronrezeptor und „human epidermal growth factor receptor 2" (HER2) beim Mammakarzinom aufgelegt. Alle Pathologie-Institute können unter www.ringversuch.de über einen Zeitraum bis zum 31.12.2011 wieder vorgetestetes Material anfordern und damit ihr eigenes Nachweisverfahren überprüfen. Das bewährte Verfahren unterscheidet sich nicht von dem der vorangegangenen Jahre.

Das 10-jährige Jubiläum einer Institution, die in der deutschsprachigen $\mathrm{Pa}$ thologie vorher nicht existent war, bildet einen Anlass, das Erreichte einer kritischen Würdigung zu unterziehen und einen Blick in die Zukunft zu werfen. Den Ausgangspunkt bildete im Jahr 2000 eine Initiative des Würzburger Instituts unter Prof. H.K. Müller-Hermelink und unter wesentlicher Beteiligung von Prof. T. Rüdiger, zu der andere Institute aus München (Prof. Höfler), Rostock (Prof. Nizze), Bonn (Prof. Pfeifer), Berlin (Prof. Stein) und Hannover (Medizinische Hochschule Hannover, $\mathrm{MHH}$ ) beitrugen. Es wurde ein Ringversuch geplant, der die Integration der Immunhistochemie in den diagnostischen Prozess abbilden sollte.

Bereits bei der Konzipierung wurde kontrovers diskutiert, ob nicht mit dem Versuch, die tägliche Praxis in der Immunhistochemie (Indikationsstellung, Auswahl der Antikörper, Färbung und Auswertung) abzubilden, die histologische Diagnose ein zu starkes Gewicht be- käme und eher hierüber als über die technischen Aspekte der Immunhistochemie ein Vergleich stattfände.

Als Kompromiss wurde, weltweit erstmalig, ein "tissue microarray" eingeschlossen, bei dem es nur um die Frage der Hormonrezeptordarstellung beim Mammakarzinom ging. Günstige Voraussetzung hierfür war, dass im Institut für Pathologie der MHH durch R. Lilischkis, M. Mengel und R. von Wasielewski ein neues Verfahren der Tissue-microarrayHerstellung entwickelt worden war, das hierfür eingesetzt werden konnte.

\section{( ) An den Ringversuchen nahmen jeweils zwischen 120 und 250 Institute für Pathologie teil}

Tatsächlich zeigte dann die Auswertung des Versuchs, an dem 172 Institute für Pathologie teilnahmen, dass die histologische Verdachtsdiagnose die alles entscheidende Variable bildete, wenn mithilfe der Immunhistochemie eine korrekte Diagnose erzielt werden sollte [1]. Der rein technische Teil des Ringversuchs mit dem Tissue microarray erbrachte allerdings auch, dass mit diesem Instrument die Sicherheit eines immunhistochemisch basierten quantitativen Nachweisverfahrens effektiv überprüft werden kann [2]. Später bedienten sich auch andere Qualitätssicherungsmaßnahmen, darunter in den USA, dieses Instruments [3].

Während der diagnostisch ausgerichtete Teil des Ringversuches aus den genannten Gründen nicht fortgesetzt wur- de, geschah dies mit dem mehr technisch orientierten Teil, der sich auf Tissue Microarrays stützte und bei dem es nicht um die Stellung einer Diagnose, sondern um die Bestimmung einer tumorbiologischen Eigenschaft ging. Von diesem Jahr an mit einer Unterbrechung wurden jährlich Ringversuche zum Nachweis von Östrogen- und Progesteronrezeptoren sowie HER2 angeboten, an denen jeweils zwischen 120 und 250 Institute für Pathologie teilnahmen. Außerdem wurden wegen der herausragenden Bedeutung für die Graduierung des Mammakarzinoms Ringversuche für Ki-67 durchgeführt [4].

Im Jahr 2002 ergriff die Deutsche Gesellschaft für Pathologie die Initiative und forderte Institute für Pathologie, die hinsichtlich eines bestimmten immunhistochemischen oder molekularpathologischen tumorbiologischen Parameters über eine besondere wissenschaftliche Expertise verfügten, auf, sich als Referenzinstitution zur Verfügung zu stellen. Jeweils 3 solcher Referenzinstitute sollten ein Panel bilden und für die Auswahl und Vortestung von Ringversuchsproben sowie die Festlegung der Bestehensregeln verantwortlich sein. Neben c-kit, Mikrosatelliteninstabilitätsmarkern, Tuberkulose-Polymerase-Kettenreaktion u. a. wurde auch ein Panel für die Testung der tumorbiologischen Parameter beim Mammakarzinom ins Leben gerufen. $\mathrm{Zu}$ diesem gehören ein Praxisinstitut (Kassel), ein Krankenhausinstitut (Wiesbaden) und ein Universitätsinstitut (Hannover). 
Das Panel hat die Aufgabe, geeignetes Probenmaterial zu selektieren und vorzutesten, sodass nur standardisiertes Material, das unabhängig vom eingesetzten Antikörper zu einem einheitlichen Untersuchungsergebnis führt, in den Ringversuch eingeht. Hierzu sind umfangreiche Vortestungen nötig.

Neben der Materialauswahl bestimmt das Panel auch über die Strukturmerkmale des Ringversuchs. Hierzu gehört die gesonderte Betrachtung von Laborund Auswerteergebnis, da eine richtige Anfärbung falsch ausgewertet und eine falsche Färbung richtig ausgewertet werden kann. Diese Differenzierung, die sich nicht auf die einfache Mitteilung, richtig oder falsch, beschränkt, erklärt sich aus der grundlegenden Intention des Ringversuchs, der nicht selektiv, sondern informativ wirken will. Dazu werden alle Färbungen jeder Teilnehmer nachbegutachtet und mit einer Stellungnahme versehen; je nach Teilnehmerzahl sind dies bis $\mathrm{zu} 23.000$ Immunfärbungen/Ringversuch.

Über das erfolgreiche Bestehen eines Ringversuchs entscheidet auch die Auswahl des Materials mit. Werden überwiegend schwere Fälle angeboten, ist die Zahl der Nichtbesteher deutlich höher, als wenn nur eindeutige Proben eingeschlossen werden. Andererseits sind gerade die diffizilen Grenzfälle geeignet, über latente Schwächen im eigenen Labor Aufschluss zu geben.

Dem Zielkonflikt zwischen selektiver und informativer Intention des Ringversuchs wurde dadurch begegnet, dass es einen bestehensrelevanten „Testteil“ gibt, in dem ausschließlich Fälle vertreten sind, deren Zusammensetzung die Alltagswirklichkeit widerspiegelt, und einen „Trainingsteil“, in dem schwierige Grenzfälle angereichert und damit überrepräsentiert sind. Unterdurchschnittliches Abschneiden im Trainingsteil wird lediglich mitgeteilt und entscheidet nicht über die Vergabe des Zertifikats. Abweichungen müssen bewertet und in ihrer Schwere eingeordnet werden, da eine Beurteilung HER2 $1+$ bei einem Zielwert von o weniger gravierend ist als bei einem Zielwert von $2+$. Dazu wurde ein Punktesystem mit maximal 3 Punkten/Probe entwickelt. Dieses System wurde von anderen Ringversu- chen, z. B. für K-ras, übernommen (hier mit 2 Punkten).

Natürlich muss sich nach 10 Jahren ein Qualitätssicherungsinstrument, das nicht nur zur Vergabe von dekorativen Urkunden, sondern zur Optimierung der Versorgungssituation von Patientinnen mit Brustkrebs eingerichtet wurde, der Frage stellen, ob tatsächlich Verbesserungen eingetreten sind. Folgende Beobachtungen sprechen dafür, dass dies tatsächlich der Fall ist: Es konnte gezeigt werden, dass eine wiederholte Teilnahme am Ringversuch mit einem verbesserten Färbe- und Auswerteergebnis einherging [5]. Offensichtlich führten die Informationen über Schwächen im eigenen Nachweisverfahren zu einer Anpassung und Verbesserung der Methodik [5].

\section{(7) Die wiederholte Teilnahme am Ringversuch ging mit einem verbesserten Ergebnis einher}

Bei einer neoadjuvanten Therapiestudie, die in 5. Auflage durchgeführt wird und bei der präoperativ mit Trastuzumab behandelt wird, zeigte sich erstmalig kein großer Unterschied mehr im Ansprechen zwischen dem zentral und dezentral bestimmten HER2-Status (G. von Minckwitz, pers. Mitteilung). Im HER2-Monitor wurden über 23.00o Bestimmungen aus 49 Instituten in Deutschland, der Schweiz und Österreich erfasst [6]. Dabei stellte sich ein Mittelwert von 16\% HER2positiver Fälle pro Institut mit nur wenigen Abweichungen heraus. Auch ergab sich, dass es bei Abweichungen eher zu einer Unter- als zu einer Überschätzung des Anteils HER2-positiver Fälle kommt. Interessanterweise hatte ein Teil der Institute, die außerhalb des 99,5\%-Konfidenzintervalls lagen, in zumindest einem Ringversuch (Test- und Trainingsteil) die Information erhalten, dass die Sensitivität ihres Nachweisverfahrens zu gering ist. Dies spricht dafür, dass der Ringversuch, so wie er angelegt ist, über latente oder manifeste Schwächen im Nachweisverfahren, die auf die Dauer zu einer signifikanten Abweichung der Positivitätsrate führen, Auskunft geben kann.

Ein Blick in die Zukunft zeigt Herausforderungen, die weiter zu bestehen oder noch zu lösen sind. Die drei Hauptaufga- ben sind mit den Schlagworten Vertrauensschutz, Interdisziplinarität und Europäisierung zu umreißen.

Anders als die Labormedizin sind die Institute für Pathologie nicht per Gesetz zur Teilnahme an Ringversuchen verpflichtet, auch wenn Zertifizierungsleitlinien der Deutschen Krebsgesellschaft entsprechende Teilnahmen einfordern. Dennoch bleibt die Exposition gegenüber der Gefahr des Nichtbestehens in einem Ringversuch die grundsätzlich freiwillige Entscheidung eines Instituts, das damit auch sein vitales Interesse an einer Qualitätsoptimierung unter Beweis stellt. Hierfür muss es unbedingten Vertrauensschutz genießen, und negative Teilnahmeergebnisse dürfen bei ubiquitärer Kompetition gerade in diesem Sinn nicht instrumentalisierbar werden. Hier haben die Organisatoren von Ringversuchen eine besondere Treuhänderfunktion, und Verschwiegenheit muss gewahrt werden, wenngleich positive Bestehenserfolge selbstverständlich kommunizierbar bleiben müssen. In den 10 Jahren des Ringversuchs ist es in keinem einzigen Fall zu einer Beschwerde wegen eines potenziellen Bruchs der Vertraulichkeit gekommen.

Große Bedeutung haben die Ringversuche auch gegenüber den kooperierenden klinischen Disziplinen, die sich darauf verlassen können müssen, dass aus fachinternen oder berufspolitischen Gründen Qualitätsstandards nicht relativiert, sondern an dem Bedarf der kooperierenden Disziplinen ausgerichtet werden. Gerade wegen des interdisziplinären Aspekts unterstützt die Deutsche Krebsgesellschaft als zuständige interdisziplinäre Fachgesellschaft für die Krebsbehandlung wieder den Ringversuch zum Mammakarzinom 2011/2012, wie auch die in den Jahren zuvor.

Im zusammenwachsenden Europa stellt sich die Frage "Zentralisieren oder Standardisieren“ bei der Bestimmung tumorbiologischer Parameter aufs Neue. Dies gilt insbesondere, da viele Länder überhaupt noch nicht über eine entsprechend methodisch breit aufgestellte Pathologie, wie in Deutschland, der Schweiz und Österreich, verfügen. Die European Society of Pathology hat daher die Qualitätssicherung insbesondere in 
der Molekularpathologie zu ihrem Anliegen gemacht. Dabei wird es nicht darum gehen, ein komplett neues System zu erschaffen, sondern in Europa bereits etablierte Systeme für alle Institute für $\mathrm{Pa}$ thologie in Europa zugänglich zu machen und Mindeststandards zu definieren. Mit den Worten von Keith Miller, dem Direktor der Sektion für Immunhistochemie und In-Situ-Hybridisierung innerhalb des United Kingdom National External Quality Assessment Service (UK NEQAS):

I understand that there is broad agreement to have regional molecular schemes with some form of central coordination/accreditation. From experience, this would make perfect sense. The European economic area as a whole is much too large to have one scheme covering all countries. Perhaps only three or four schemes would be required. This would ensure that each would be sufficiently large enough so that they would be economically viable.

Für die Institute für Pathologie in Europa bedeutet dies, dass sie in Zukunft eine größere Auswahl und einen internationalen Vergleich haben, was Qualitätssicherungsmaßnahmen angeht, und für die Ringversuche im deutschsprachigen Raum, dass sie europäischen Standards entsprechen müssen, um auch in Zukunft zu bestehen.

\section{Korrespondenzadresse}

\section{Prof. Dr. H.H. Kreipe}

Institut für Pathologie,

Medizinische Hochschule Hannover

Carl-Neuberg-Str. 1, 30625 Hannover

kreipe.hans@mh-hannover.de

Interessenkonflikt. Der korrespondierende Autor gibt an, dass kein Interessenkonflikt besteht.

\section{Literatur}

1. Rüdiger T, Höfler H, Kreipe HH et al (2002) Quality assurance in immunohistochemistry: results of an interlaboratory trial involving 172 pathologists. Am J Surg Pathol 26:873-882

2. von Wasielewski R, Mengel M, Wiese B et al (2002) Tissue array technology for testing interlaboratory and interobserver reproducibility of immunohistochemical estrogen receptor analysis in a large multicenter trial. Am J Clin Pathol 118:675-682
3. Dorfman DM, Bui MM, Tubbs RR et al; College of American Pathologists Cell Markers Committee (2006) The CD117 immunohistochemistry tissue microarray survey for quality assurance and interlaboratory comparison: a College of American Pathologists Cell Markers Committee Study. Arch Pathol Lab Med 130:779-782

4. Mengel M, von Wasielewski R, Wiese B et al (2002) Inter-laboratory and inter-observer reproducibility of immunohistochemical assessment of the Ki-67 labelling index in a large multi-centre trial. J Pathol 198:292-299

5. Wasielewski R, Hasselmann S, Rüschoff J et al (2008) Proficiency testing of immunohistochemical biomarker assays in breast cancer. Virchows Arch 453:537-543

6. Choritz H, Büsche G, Kreipe H (2011) Quality assessment of HER2 testing by monitoring of positivity rates. Virchows Arch, doi 10.1007/ s00428-011-1132-8

\section{Nierentumoren}

In den vergangenen 10 Jahren nahm die Inzidenz des Nierenzellkarzinoms erheblich zu. Obwohl heute eine frühzeitigere Diagnose möglich ist, hat sich die Prognose der Erkrankung jedoch kaum verbessert. Zahlreiche Studien belegen, dass das Entfernen von Nierengewebe und die damit einhergehende Verschlechterung der Nierenfunktion einen entscheidenden Einfluss auf die Prognose der Patienten haben. Durch eine differenzierte operative bzw. eine zielgerichtete medikamentöse Therapie sollte die Prognose des Nierenzellkarzinoms verbessert und die Nierenfunktion der betroffenen Patienten möglichst lange Der Nephrologe

$=$ erhalten werden. Der aktuelle Stand zu Diagnostik, Pathogenese und Therapie des Nierenzellkarzinoms ist im Leitthemenheft "Nierentumoren" (Ausgabe 4/2011) von „Der Nephrologe" u. a. in folgenden Beiträgen dargestellt:

- Diagnostik des Nierenzellkarzinoms

- Pathologie und molekulare Pathogenese des Nierenzellkarzinoms

- Operative Therapie des Nierenzellkarzinoms

- Systemtherapie des fortgeschrittenen Nierenzellkarzinoms

- Bedeutung der Nierenfunktion für die Therapiestrategien des Nierenzellkarzinoms

Bestellen Sie diese Ausgabe zum Preis von EUR 34,- zzgl. Versandkosten bei:

Springer Customer Service Center $\mathrm{GmbH}$ Kundenservice Zeitschriften

Haberstr. 7

69126 Heidelberg

Tel.: +49 6221-345-4303

Fax: +49 6221-345-4229

leserservice@springer.com

www.dernephrologe.de

P.S. Vieles mehr rund um Ihr Fachgebiet finden Sie auf www.springermedizin.de 